\title{
COMPLICACIONES MATERNO-NEONATALES EN PACIENTES CON DIABETES GESTACIONAL, HOSPITAL GINECO-OBSTETRICO ENRIQUE C. SOTOMAYOR
}

\author{
MATERNAL-NEONATAL COMPLICATIONS IN PATIENTS WITH GESTATIONAL DIABETES AT THE \\ ENRIQUE C. SOTOMAYOR OB-GYN HOSPITAL
}

\author{
COMPLICAÇ̃̃ES MATERNO-NEONATAIS EM PACIENTES COM DIABETES GESTACIONAL NO HOSPITAL GINECO- \\ OBSTETRICO ENRIQUE C. SOTOMAYOR
}

\author{
PAULINA DASSUM AMADOR', ADRIANA SERRANO SALGADO', CONRAD RIEMANN TORRES ${ }^{1}$ \\ ${ }^{1}$ Universidad Católica de Santiago de Guayaquil, Guayaquil, Ecuador
}

\begin{abstract}
Introducción: la diabetes gestacional es la complicación metabólica más frecuente del embarazo afectando entre el 3-10\% de las gestantes. Los hijos de gestantes diabéticas tienen mayor riesgo de presentar complicaciones perinatales como la hipoglucemia, prematurez, macrosomía, síndrome de dificultad respiratoria, malformaciones congénitas e incluso muerte neonatal. Objetivo: determinar si existe diferencia en relación a las complicaciones gestacionales en pacientes con y sin diabetes gestacional. Materiales y métodos: se realizó un estudio transversal, en el que se compararon las medidas antropométricas del recién nacido (peso, talla, semana gestacional, puntaje Apgar) y las complicaciones materno-neonatales (muerte materna, malformaciones congénitas, distrés respiratorio neonatal, óbito fetal) entre pacientes embarazadas con diagnóstico de diabetes gestacional y otro grupo sin diabetes gestacional en el hospital Gineco-Obstétrico Enrique C. Sotomayor. Resultados: se estudiaron 180 pacientes, el promedio de la edad materna fue de $30.2 \pm 5.9$ años. Se compararon 90 pacientes con diagnóstico de diabetes gestacional con 90 pacientes sin este diagnóstico. Las complicaciones maternas fueron más frecuentes en las mujeres con diabetes gestacional ( $n=52$ vs. 27, valor $p<0.01$ ), los trastornos hipertensivos en el embarazo ( $n=19$ vs. 9, valor $\mathrm{p}: 0.03$ ) y el polihidramnios ( $\mathrm{n}: 8 \mathrm{vs}$. 2, valor $\mathrm{p}$ : 0.05). El número de óbitos fetales y la mortalidad neonatal se presentó con mayor frecuencia en el Grupo 1 ( $n=6$ vs. $0, p: 0.01 ; n: 8$ y 1, p: 0.01; respectivamente). Las complicaciones metabólicas neonatales, el distrés respiratorio y las malformaciones congénitas se presentaron con mayor frecuencia en el Grupo 1. Conclusión: se encontró mayor frecuencia de morbimortalidad maternoneonatal en mujeres con diabetes gestacional, por lo que se recomienda: tomar medidas preventivas a nivel comunitario para evitar el desarrollo de la diabetes gestacional, realizando controles rigurosos de glicemia prenatales rigurosos.
\end{abstract}

PALABRAS CLAVE: diabetes gestacional, complicaciones del embarazo, neonato, morbimortalidad.

Introduction: gestationaldiabetesis the most frequentmetabolic complication of pregnancy affe diadiction: gestational diabetes is the most frequent metabolic complication of pregnancy aftecting between $3-10 \%$ of pregnant women. Children of syndrome, congenital malformations and even neonatal death. Objective: to determine if there is a difference regarding gestational complications in patients with and without gestational diabetes. Materials and methods: cross-sectional study was carried out, comparing the anthropometric measurements of the newborn (weight, height, gestational week, Apgar score) and maternal-neonatal complications (maternal death, congenital malformations, neonatal respiratory distress, death). fetal) among pregnant patients diagnosed with gestational diabetes and another group without gestational diabetes at the Enrique C. Sotomayor Obstetric-Gynecological hospital. Results: 180 patients were studied. Average maternal age was $30.2 \pm 5.9$ years. 90 patients diagnosed with gestational diabetes were compared to 90 patients without this diagnosis. Maternal complications were more frequent in women with gestational diabetes $(n=52$ vs. 27, p value $<0.01$ ), hypertensive disorders in pregnancy $(n=19$ vs. $9, p$-value: 0.03 ) and polyhydramnios $(n: 8$ vs. 2 , $\mathrm{p}$-value: 0.05 ). The number of fetal deaths and neonatal mortality occurred more frequently in Group 1 ( $n=6 \mathrm{vs.} 0, \mathrm{p}: 0.01, \mathrm{n}: 8$ and 1, p: 0.01, respectively). Neonatal metabolic complications, respiratory distress and congenital malformations occurred more frequently in Group 1. Conclusion: higher frequency of maternal-ononatal morbidity and mortality was found in women with gestational diabetes, so it is recommended to take preventive measures such as rigorous prenatal blood glucose controls so as to inhibit development of the disease.

KEYWORDS: diabetes, gestational, pregnancy complications, newbor, morbidity and mortality

Resumo

Introdução: a diabetes gestacional é a complicação metabólica mais frequente da gravidez afetando entre $03-10 \%$ das mulheres gravidas. Os filhos de gestantes diabéticas têm maior risco de apresentar complicações perinatais como hipoglicemia, prematuridade, microssomia, síndrome de dificuldade respiratória, malformações congênitas até morte neonatal. Objetivo: determinar se tem diferença em relação as complicações gestacionais em pacientes come sem diabetes gestacional. Materiais e métodos: foi realizado um estudo transversal, onde são comparadas as medidas antropométricas do neonato (peso, escultura, semana gestacional, Apgar) e as complicações materno-neonatal (morte da mãe, malformações congênitas, distres respiratório neonatal, óbito fetal) entre pacientes gravidas com diagnostico de diabetes gestacional e outro grupo sem diabetes gestacional no hospital Gineco-Obstétrico Enrique C. Sotomayor. Resultados: foram estudadas 180 pacientes, a media de idade materna foi $30.2 \pm 5.9$ anos. Se compararam 90 pacientes com diagnostico de diabetes gestacional com 90 pacientes sem este diagnostico. As complicações maternas foram mais frequentes nas mulheres com diabetes gestacional ( $n=52$ vs. 27, valor $p<0.01$ ), os transtornos hipertensivos na gravidez ( $n=19$ vs. 9, valor $p: 0.03$ ) e o polihidramnios ( $n: 8$ vs. 2, valor $p: 0.05$ ). 0 numero de óbitos fetais e mortalidade neonatal se apresentou com maior frequência no grupo 1. Conclusão: se encontrou maior frequência de morbimortalidade materno-neonatais nas mulheres com diabetes gestacional. É recomendado: prevenção ao nível comunitário para evitar o desenvolvimento da mesma e controles da glicemia pré-natais rigorosos.

PALABRAS-CHAVE: diabetes gestacional, complicações na gravidez, recém-nascido, morbimortalidade. 
INTRODUCCIÓN

La diabetes gestacional (DG) es la intolerancia a los carbohidratos en diferentes grados de severidad que es diagnosticada por primera vez durante el embarazo. Se estima que aproximadamente de un 3 al $10 \%$ de embarazadas padecen de diabetes gestacional, siendo la complicación metabólica más frecuente del embarazo.

Se diagnostica diabetes gestacional si durante el embarazo se obtienen niveles de glucosa mayores de $126 \mathrm{mg} / \mathrm{dl}$ en dos ocasiones o si presenta una curva de tolerancia a la glucosa anormal.' Los factores de riesgo más reportados para DG son: antecedente personal de diabetes gestacional, que aumenta el riesgo 33-50 \%, y aún más si hay un segundo factor de riesgo. ${ }^{2}$ Otros factores descritos son la historia familiar en primer grado de diabetes mellitus (DM), edad materna (> 25 años), sobrepeso previo al embarazo (IMC > $25 \mathrm{~kg} / \mathrm{m} 2$ ), antecedente obstétrico adverso (mortinatos, peso fetal > $4000 \mathrm{~g}$ ), multiparidad (tres o más hijos). ${ }^{2} \mathrm{El}$ riesgo aumenta cuando múltiples factores de riesgo se presentan conjuntamente. ${ }^{3}$

El interés por esta patología ha aumentado debido al pronóstico adverso que presenta tanto la madre como el feto. La DG altera diversos sistemas en el feto, el pobre control glicémico desde el inicio del embarazo afecta la organogénesis y el descontrol tardío afecta el desarrollo corporal. ${ }^{4}$

Los hijos de madres con diabetes gestacional tienen mayor riesgo de presentar complicaciones metabólicas asociadas como hipoglucemia ademas de prematurez, macrosomía, síndrome de dificultad respiratoria, malformaciones congénitas e incluso la muerte neonatal. ${ }^{5} \mathrm{La}$ prevalencia de la DG a nivel mundial en poblaciones de bajo riesgo oscila entre el 1,4\% y el 2,8 $\%$, mientras que en poblaciones de alto riesgo entre el 3,3\% y el 6,1\%, presentando variación según la etnia. ${ }^{6,7,8}$

En el Ecuador no se conocen datos epidemiológicos que demuestren la prevalencia de la patología o sus complicaciones neonatales. Sin embargo, de acuerdo a la ENSANUT (Encuesta Nacional de Salud y Nutrición) $48.3 \%$ de mujeres en edad reproductiva entre 12 y 49 años padecen de sobrepeso y obesidad. ${ }^{9}$ El objetivo primario de este estudio es comparar la frecuencia de las complicaciones materno-neonatales en pacientes embarazadas con diagnóstico de diabetes gestacional, respecto a aquellas pacientes sin este diagnóstico.

\section{MATERIALES Y MÉTODOS}

Se realizó un estudio transversal, en el que se compararon las complicaciones maternoneonatales entre las embarazadas con diagnóstico de DG contra un grupo de embarazadas sin DG. Para el registro de los datos se exploraron las historias clínicas del hospital gineco-obstétrico Enrique C. Sotomayor desde noviembre de 2013 hasta noviembre de 2014

Los criterios de inclusión para el primer grupo fuero (a) pacientes con diagnóstico de DG definida como: nivel de glucosa mayor a $126 \mathrm{mg} /$ $\mathrm{dl}$ en dos ocasiones o una curva de tolerancia a la glucosa anormal después de una carga de glucosa en ayuno de $100 \mathrm{~g}$, definida como: un valor superior a $190 \mathrm{mg} / \mathrm{dl}$ una hora después de recibir la carga, o $165 \mathrm{mg} / \mathrm{dl}$ a las dos horas post carga, o $145 \mathrm{mg} / \mathrm{dl}$ a las tres horas post carga; (b) que cuenten con historias clínicas maternas y neonatales completas. Los criterios de exclusión fueron: (a) pacientes embarazadas con diagnóstico previo de diabetes tipo I o II; (b) que presenten comorbilidades diagnosticadas previo al embarazo: lupus eritematoso sistémico, insuficiencia renal, hepática o cardiaca, entre otras; (c) historia de ingesta de fármacos o productos teratógenos durante el embarazo. El grupo comparación incluyó pacientes embarazadas sin diagnóstico de DG que contaran con historias clínicas maternas y neonatales completas.

Debido a limitaciones temporales se realizó un estudio con muestreo no probabilístico consecutivo. Las pacientes no se aparearon por ningún criterio entre los dos grupos.

Se obtuvieron como variables a la glicemia preparto/precesárea en $\mathrm{mg} / \mathrm{dl}$; el peso del recién nacido en gramos y su evaluación clínica, según la edad gestacional (AEG, PEG, GEG), la talla del recién nacido en centímetros, la edad gestacional del neonato según la escala de Ballard; y, el estado general del recién nacido según el puntaje Apgar al minuto y a los cinco minutos, así como: las complicaciones maternas, complicaciones neonatales, óbitos fetales, aborto espontáneo, malformaciones congénitas, muerte fetal como presente o ausente. 
Adicionalmente se recolectaron las siguientes características basales de las gestantes: edad materna en años, número de gestas, partos y cesáreas, vía del nacimiento (parto vaginal o cesárea), así como los factores de riesgo para el desarrollo de DG como presentes o ausentes (familiares diabéticos de primer grado, obesidad, óbitos fetales previos, DG en embarazo previo).

Para el análisis estadístico se reportan las variables cuantitativas continuas como promedio y desviación estándar; en estas variables se utilizó la prueba t de Student para calcular el valor p. Las variables cualitativas se presentaron como frecuencias simples y porcentajes. Para obtener el valor p se usó la prueba de Chi cuadrado.

Se consideró resultados estadísticamente significativos los que presentaron un valor $\mathrm{p}<0.05$. La información requerida se la extrajo de los datos del programa MIS de la base de datos del Hospital, exportado a una hoja de Microsoft Office Excel 2007 versión para Windows ${ }^{\circledR}$ y se utilizó el programa estadístico MedCalc ${ }^{\circledR}$ 12.7.5. Para el presente estudio se siguieron las recomendaciones de la declaración de Helsinki.

\section{RESULTADOS}

Cumplieron con los criterios de inclusión y exclusión 180 pacientes, el promedio de edad materna fue de $30.2 \pm 5.9$ años. Presentaron diagnóstico de DG 90 pacientes (grupo 1); y 90 pacientes no presentaron diagnóstico de DG (grupo 2).

En la tabla 1, se resumen las características basales de cada grupo; de ellas la edad materna (32.8 años \pm 5.9 vs. 27.6 años $\pm 4.6, p<0.01$ ), factores de riesgo como: familiar diabético $(\mathrm{n}=$ 28 vs. $8, p<0.01$ ), obesidad ( $n=10$ vs. $0, p<0.01$ ) y la ausencia de los mismos ( $\mathrm{n}=41$ vs. 80 , $\mathrm{p}$ $<0.01$ ) mostraron diferencias estadísticamente significativas.

En los antecedentes gineco-obstétricos, se encontró $\geq 3$ partos ( $n=39$ vs. 24 , valor $p=0.01$ ); sin antecedente de partos ( $\mathrm{n}=61$ vs. 36 , valor $\mathrm{p}<0.01$ ); abortos $\geq 3$ ( $\mathrm{n}=6$ vs. 1 , valor $\mathrm{p}=0.05$ ); cesárea $\geq 3$ ( $n=9$ vs. 2 , valor $p=0.03$ ). En el grupo 2 entre las diferencias significativas se encontraron: gestas 2 ( $\mathrm{n}=16$ vs. 39 , valor $\mathrm{p}=0.01$ ); parto 1 ( $\mathrm{n}=8$ vs. 23 , valor $\mathrm{p}=0.003)$, partos $2(\mathrm{n}=$ 9 vs. 19 , valor $\mathrm{p}=0.03)$ y ninguna cesárea $(\mathrm{n}=41$ vs. 58 , valor $\mathrm{p}=0.01$ ).
TABLA 1. CARACTERÍSTICAS BASALES DE LOS DOS GRUPOS DE ESTUDIO

\begin{tabular}{lrrr}
\hline VARIABLES & $\begin{array}{r}\text { GRUPO 1 } \\
\text { N=90 (\%) }\end{array}$ & \multicolumn{1}{c}{$\begin{array}{c}\text { GRUPO 2 } \\
\text { N=90 (\%) }\end{array}$} & VALOR P \\
\hline Edad materna & $32.8 \pm 5.9$ & $27.6 \pm 4.6$ & $<0.01$ \\
Factores de riesgo & & & \\
DG & $3(3.3)$ & $0(0)$ & 0.08 \\
DG y familiar diabético & $1(1.1)$ & $0(0)$ & 0.31 \\
Familiar diabético & $28(31.1)$ & $8(8.9)$ & $<0.01$ \\
Obesidad & $10(11.1)$ & $0(0)$ & 0.001 \\
Obesidad y familiar & $3(3.3)$ & $0(0)$ & 0.08 \\
diabético & & & \\
Óbito fetal & $0(0)$ & $2(2.2)$ & 0.15 \\
Ninguno & $41(45.7)$ & $80(88.9)$ & $<0.01$
\end{tabular}

\begin{tabular}{|c|c|c|c|}
\hline \multicolumn{4}{|c|}{$\begin{array}{l}\text { Antecedentes gineco-obstétricos } \\
\text { Gestas }\end{array}$} \\
\hline 0 & $15(16.7)$ & $9(10)$ & 0.18 \\
\hline 1 & $20(22.2)$ & $18(20)$ & 0.71 \\
\hline 2 & $16(17.8)$ & $39(43.3)$ & $<0.01$ \\
\hline$\geq 3$ & $39(43.3)$ & $24(26.7)$ & 0.01 \\
\hline \multicolumn{4}{|l|}{ Partos } \\
\hline 0 & $61(67.8)$ & $36(40)$ & $<0.01$ \\
\hline 1 & $8(8.9)$ & $23(25.6)$ & 0.003 \\
\hline 2 & $9(10)$ & 19 (21.1) & 0.03 \\
\hline$\geq 3$ & $12(13.3)$ & $12(13.3)$ & $>0.05$ \\
\hline \multicolumn{4}{|l|}{ Abortos } \\
\hline 0 & $53(58.9)$ & $62(68.9)$ & 0.16 \\
\hline 1 & $26(28.9)$ & $21(23.3)$ & 0.39 \\
\hline 2 & $5(5.5)$ & $6(6.7)$ & 0.75 \\
\hline$\geq 3$ & $6(6.7)$ & $1(1.1)$ & 0.05 \\
\hline \multicolumn{4}{|l|}{ Cesáreas } \\
\hline 0 & $41(45.5)$ & $58(64.4)$ & 0.01 \\
\hline 1 & $26(28.9)$ & $17(18.9)$ & 0.11 \\
\hline 2 & $14(15.6)$ & $13(14.4)$ & 0.83 \\
\hline$\geq 3$ & $9(10)$ & $2(2.2)$ & 0.03 \\
\hline $\begin{array}{l}\text { Glicemia preparto/ } \\
\text { cesárea }\end{array}$ & $116.8 \pm 40.9$ & $86.3 \pm 10.5$ & $<0.01$ \\
\hline \multicolumn{4}{|l|}{ Vía del nacimiento } \\
\hline Parto vaginal & $2(2.2)$ & $43(47.8)$ & $<0.01$ \\
\hline Cesárea & $88(97.8)$ & $47(52.2)$ & 0.02 \\
\hline
\end{tabular}

La glicemia preparto/cesárea fue mayor en las pacientes del grupo $1(116.8 \mathrm{mg} / \mathrm{dl} \pm 40.9$ vs. $86.3 \mathrm{mg} / \mathrm{dl} \pm 10.5, \mathrm{p}<0.01)$. En el grupo 1 la vía de parto por cesárea fue mayor que en el grupo 2 ( $\mathrm{n}=88$ vs. 47, valor $\mathrm{p}<0.02$ ). Se observó que el grupo 2 tuvo mayor cantidad de partos vaginales en comparación con el grupo 1 ( $\mathrm{n}=43$ vs. 2, valor $\mathrm{p}<0.01$ ).

Se observó que la edad gestacional de los neonatos fue menor en el grupo 1 ( $37 \mathrm{SC} \pm 3$ vs. $38.1 \mathrm{SC}$ \pm 1.9 , valor $\mathrm{p}<0.01$ ), así también la puntuación APGAR al minuto $(6.5 \pm 2$ vs. $7.7 \pm 0.8$, valor $p$ $<0.01$ ), a los 5 minutos ( $7.05 \pm 1.95$ vs. $8.2 \pm 0.68$, valor $\mathrm{p}<0.01)$ y la talla de los neonatos $(46.4 \mathrm{~cm} \pm$ 
4.2 vs. $47.7 \mathrm{~cm} \pm 3.4$, valor $\mathrm{p}=0.01$ ) (tabla 3). En la evaluación del peso gestacional, se encontró una mayor frecuencia de neonatos grandes para su edad gestacional ( $\mathrm{n}=22$ vs. 7 , valor $\mathrm{p}=0.001$ ) en el grupo 1. (Tabla 2).

TABLA 2. RESULTADOS ANTROPOMÉTRICOS Y APGAR SEGÚN LOS GRUPOS DE ESTUDIO

\begin{tabular}{|c|c|c|c|}
\hline VARIABLES & GRUPO $1 \mathrm{~N}=90$ & GRUPO 2 N=90 & VALOR P \\
\hline Edad gestacional & $37 \pm 3$ & $38.1 \pm 1.9$ & $<0.01$ \\
\hline \multicolumn{4}{|l|}{ APGAR } \\
\hline 1 minuto & $6.5 \pm 2$ & $7.7 \pm 0.8$ & $<0.01$ \\
\hline 5 minutos & $7.05 \pm 1.95$ & $8.2 \pm 0.68$ & $<0.01$ \\
\hline Peso (g) & $3012.8 \pm 824.8$ & $2932 \pm 574.4$ & 0.44 \\
\hline \multicolumn{4}{|c|}{ Evaluación peso gestacional } \\
\hline $\mathrm{AEG}^{\mathrm{a}}$ & $66(73.4)$ & $76(84.4)$ & 0.31 \\
\hline $\mathrm{GEG}^{\mathrm{b}}$ & $22(24.4)$ & $7(7.8)$ & 0.001 \\
\hline $\mathrm{PEG}^{\mathrm{C}}$ & $2(2.2)$ & $7(7.8)$ & 0.10 \\
\hline Talla (cm) & $46.4 \pm 4.2$ & $47.7 \pm 3.4$ & 0.01 \\
\hline
\end{tabular}

aAEG: adecuado para edad gestacional, bGEG: grande para edad gestacional cPEG: pequeño para edad gestacional

Las complicaciones maternas fueron más frecuentes en el grupo 1 ( $n=52$ vs. 27, valor $\mathrm{p}<0.01$ ). Los trastornos hipertensivos en el embarazo $(\mathrm{n}=$ 19 vs. 9 , valor $\mathrm{p}=0.03)$ y el polihidramnios $(\mathrm{n}=8$ vs. 2, valor $\mathrm{p}=0.05$ ) fueron más comunes en el Grupo 1, mientras que el desgarro perianal ( $\mathrm{n}=$ 0 vs. 4 , valor $\mathrm{p}=0.04$ ) fue más común en el grupo 2. Se observó que el número de óbitos fetales y la mortalidad neonatal se presentó con mayor frecuencia en el grupo 1 ( $n=6$ vs. 0 , valor $\mathrm{p}=0.01$; $\mathrm{y} \mathrm{n}=8$ vs. 1 , valor $\mathrm{p}=0.01$; respectivamente).

Las complicaciones metabólicas neonatales, el distrés respiratorio y las malformaciones congénitas se presentaron con mayor frecuencia en el grupo 1 ( 22 vs. $5 ; n=28$ vs. 6 ; $y$ n= 21 vs. 2 , todos con valor $\mathrm{p}<0.01$; respectivamente) (figura 1 ). De las complicaciones metabólicas, la hipoglicemia tuvo una diferencia significativa entre ambos grupos ( $n=11$ vs. 0 , valor $p<0.01$ ).

Se realizó un subanálisis de las pacientes con diagnóstico de DG (grupo 1), se dividió a las

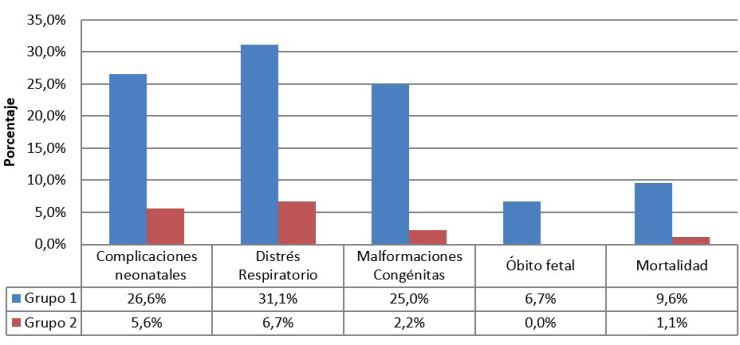

Figura 1. Eventos adversos neonatales, grupo 1 vs. grupo 2. pacientes entre las que recibieron medicación para control de glicemia y las que no. Las malformaciones congénitas fueron más frecuentes en grupo con medicación ( $n=14$ vs. 7 , valor $\mathrm{p}=0.04)$. (Tabla 3 ).

TABLA 3. COMPLICACIONES MATERNAS Y NEONATALES EN PACIENTES QUE RECIBIERON MEDICACIÓN PARA EL CONTROL DE GLICEMIA VS. PACIENTES QUE NO RECIBIERON

\begin{tabular}{|c|c|c|c|}
\hline VARIABLES & $\begin{array}{r}\text { GRUPO CON } \\
\text { MEDICACIÓN } \\
\mathbf{N}=\mathbf{4 3}\end{array}$ & $\begin{array}{r}\text { GRUPO SIN } \\
\text { MEDICACIÓN } N=47\end{array}$ & VALOR P \\
\hline $\begin{array}{l}\text { Complicaciones } \\
\text { neonatales }\end{array}$ & $9(21)$ & $13(27.6)$ & 0.45 \\
\hline Distrés respiratorio & $12(28)$ & $16(34)$ & 0.52 \\
\hline $\begin{array}{l}\text { Malformaciones } \\
\text { congénitas }\end{array}$ & $14(15.6)$ & $7(14.9)$ & 0.04 \\
\hline Óbito fetal & $2(4.6)$ & $4(8.5)$ & 0.46 \\
\hline $\begin{array}{l}\text { Complicaciones } \\
\text { maternas }\end{array}$ & $21(48.8)$ & $28(59.6)$ & 0.89 \\
\hline Mortalidad & $4(9.3)$ & $4(8.5)$ & 0.30 \\
\hline
\end{tabular}

DISCUSIÓN

Esta investigación demuestra que las complicaciones materno-neonatales se presentan con mayor frecuencia en el grupo de las madres con diabetes gestacional, entre éstas: la mortalidad neonatal, el distrés respiratorio, las complicaciones metabólicas como la hipoglicemia y en la gestante, los trastornos hipertensivos; todas con diferencias estadísticamente significativas.

De acuerdo a las caracteristicas basales, las mujeres con diabetes gestacional presentaron: un promedio mayor de edad, una frecuencia mayor de antecedentes de familiar diabético de primer grado y una mayor frecuencia de obesidad, en comparacion con las gestantes no diabéticas. La variable edad ya ha sido descrita en otros estudios mostrando resultados similares al nuestro. ${ }^{10}$ Sin embargo en otros estudios relizados por Sutton, Wright y Langer en Estados Unidos, esta diferencia puede ser menor o estadísticamente significativa. ${ }^{11,12,13}$ La historia familiar, sobretodo de primer grado, ha sido establecida hace muchos años como un factor de alto riesgo para el desarrollo de DG3. Tanto en este estudio como en el realizado en Colombia por CampoCampo et al en el año 2012, se confirma dicho riesgo. ${ }^{2}$

Es importante recordar que aunque muchos resultados siguen tendencias de acuerdo a la fisiopatología y factores de riesgo de la enfermedad, el medio donde se realiza el estudio es 
crucial. Variables como: nivel de educación, poder adquisitivo, acceso a atención médica y etnia predominante, entre otros, influyen en el desarrollo y severidad de la enfermedad. ${ }^{14,15}$

Un meta-análisis, realizado en el año 2007, reveló que las mujeres con sobrepeso, obesas o con obesidad mórbida tienen un riesgo estimado de desarrollar diabetes gestacional de dos, cuatro y ocho veces, respectivamente, comparado con las mujeres embarazadas con pesos dentro de los parámetros normales. ${ }^{16}$ De acuerdo con esta afirmación, el mayor número de pacientes obesas dentro del grupo con DG, es un riesgo potencial para el desarrollo de la patología. Además, esta afirmación pone en alerta al $48.3 \%$ de las pacientes ecuatorianas en edad reproductiva obesas o con sobrepeso, según la ENSANUT. ${ }^{9}$

El $97.8 \%$ de las pacientes con DG finalizaron el embarazo a través de operación cesárea. En otros reportes se han encontrado tasas entre el 19 y el $36.3 \%$ y el más elevado en un estudio por Velásquez et al donde la tasa fue del 41.4\%. ${ }^{11,15,16}$ Es cierto que dada la cantidad de complicaciones que presentan las madres diabéticas (macrosomía, trastornos hipertensivos, polihidramnios, etc), siguen una tendencia nacional. ${ }^{1}$

En un estudio realizado en un hospital de tercer nivel en la ciudad de Cuenca, Ecuador se encontró que la tasa de cesáreas fue del 61.6\%, siendo su principal indicación, un $32.2 \%$, la cesárea previa. ${ }^{17}$

En el presente estudio es evidente el alto número de productos GEG, en madres cesareadas. Así se ha demostrado al relacionar de manera directa las elevaciones de la glucosa sérica, con el aumento de las tasas de macrosomia y cesáreas. ${ }^{4,13,15}$ Sin embargo, los productos grandes para la edad gestacional no son enteramente atribuibles a la hiperinsulinemia, sino también a factores étnicos, edad materna, paridad, obesidad e historia de cesáreas previas. ${ }^{18,19}$

La hipoglicemia, se presentó con mayor frecuencia en el grupo de madres con DG. Se han reportado niveles bajos de glucosa en recién nacidos con una prevalencia del $5-15 \%$, en prematuros puede llegar hasta el $29 \% .20,21$ Se ha reportado que la prevalencia de hipoglicemia es más común en hijos de madres diabéticas, y podría ser que esta prevalencia sea más alta en poblaciones lantinoamericanas. ${ }^{14,22}$
Son estos motivos por los que se presentan con mayor frecuencia, complicaciones maternoneonatales en las madres con diabetes gestacional. Entre las complicaciones maternas se encontraron los trastornos hipertensivos, y el polihidramnios. Estas ya se han descrito previamente. ${ }^{23,24,25}$ Se deduce que la resistencia a la insulina, independientemente del grado de obesidad, contribuye a la hipertensión transitoria que se puede identificar en el embarazo avanzado. ${ }^{26}$

Al comparar a las pacientes con y sin medicación, sobre las malformaciones congénitas, obtuvieron frecuencias significativamente más altas en el grupo que no recibió medicación; sin embargo, la tendencia en casi todos los parámetros estudiados es mayor en el grupo de pacientes que no recibió tratamiento. Otras investigaciones han concluido que la falta de tratamiento está asociada a tasas más altas de morbilidad materna y morbimortalidad neonatal.26,27,28 Es muy importante recordar que además de los resultados a corto plazo mostrados en este estudio, los hijos de madres diabéticas gestacionales tienen un riesgo aumentado de desarrollar tanto obesidad como diabetes en etapas posteriores de la vida. ${ }^{29,30}$

Los resultados de este estudio no son concluyentes debido a la naturaleza transversal del estudio; el método de muestreo fue no aleatorio y otros factores de confusión como la edad materna avanzada junto a los trastornos hipertensivos concomitantes, pueden llevar a una sobre o subrepresentación de las complicaciones neonatales. Sin embargo, al notar que los resultados son semejantes a los encontrados en otros estudios y contar con una base fisiopatológica que pueda explicarlos, es que se cree que la población ecuatoriana se comporta semejante a las otras poblaciones o incluso de forma más severa.

\section{CONCLUSIONES}

La DG es un factor importante para la morbimortalidad materno-fetal en nuestro medio. La elevada cantidad de cesáreas contribuyen a dicha morbimortalidad pudiendo agregar el riesgo quirúrgico que conllevan. Controlar factores de riesgo como el sobrepeso y la obesidad son objetivos esenciales para disminuir posibles complicaciones. Es importante continuar el estudio de esta enfermedad investigando sus efectos a largo plazo y su asociación con los trastornos hipertensivos. 


\section{REFERENCIAS BIBLIOGRÁFICAS}

1. Velázquez, P., Vega, G., \& Martínez, M. L. Morbilidad y mortalidad neonatal asociada a la diabetes gestacional. Revista chilena de obstetricia y ginecología, 2010; 75(1), 35-41.

2. Campo-Campo M., Posada-Estrada G., BetancurBermudez L., Jaramillo-Quinceno D. Factores de riesgo para diabetes gestacional en población obstétrica en tres instituciones de Medellín, Colombia. Estudio de casos y controles. Revista Colombiana de Obstetricia y Ginecología. 2012; 63(2), 114-118.

3. Coustan DR. Diabetes mellitus in pregnancy: Screening and diagnosis. In: UpToDate, Nathan DM., Green MF. (Ed) Jan 2017.

4. Ornoy A., Reece EA., Pavlinkova G., Kappen C., Miller RK. Effect of maternal diabetes on the embryo, fetus, and children: congenital anomalies, genetic and epigenetic changes and developmental outcomes. Birth Defects Res C Embryo Today. 2015 Mar;105(1):53-72.

5. Sirimarco, M. P., Guerra, H. M., Lisboa, E. G., et al. Diagnostic protocol for gestational diabetes mellitus (GDM) (IADPSG/ADA, 2011): influence on the occurrence of GDM and mild gestational hyperglycemia $(\mathrm{MGH})$ and on the perinatal outcomes. Diabetology \& Metabolic Syndrom. 2017. 9(2).

6. Campo, M. N. C., \& Estrada, G. P. Factores de riesgo para Diabetes Gestacional en población obstétrica. CES Medicina. 2008; 22(1), 59-69.

7. Reece, E. A., Leguizamón, G., \& Wiznitzer, A. Ges tational diabetes: The need for a common ground. The Lancet. 2009; 373(9677), 1789-1797.

8. Ferrara A. Increasing prevalence of gestacional diabetes mellitus: a public health perspective. Diabetes care. 2007Jul; 30(Supplement 2): S141-S146.

9. Freire WB., Ramirez MJ., Belmont P., Mendieta MJ, Silva MK., Romero N., Saenz K., Pineiros P., Gomez LF., Monge R. 2013. RESUMEN EJECUTIVO TOMO I. Encuesta Nacional de Salud y Nutrición del Ecuador. ENSANUT-ECU 2011-2013 Ministerio de Salud Pública/Instituto Nacional de Estadística y Censos. Quito, Ecuador.

10. Baptiste-Roberts, K., Nicholson, W. K., Wang, N.-Y., \& Brancati, F. L. Gestational Diabetes and Subsequent Growth Patterns of Offspring: The National Collaborative Perinatal Project. Maternal and Child Health Journal, 2012. 16(1), 125-132.

11. Sutton AL., Mele L., Landon MB. Delivery timing and cesarean delivery risk in women with mild gestational diabetes mellitus. Am J Obstet Gynecol. 2014 Sep;211(3):244.

12. Wright, C. S., Rifas-Shiman, S. L., Rich-Edwards, J. W., Taveras, E. M., Gillman, M. W., \& Oken, E. Intrauterine Exposure to Gestational Diabetes,
Child Adiposity, and Blood Pressure. American Journal of Hypertension. 2009. 22(2), 215-220.

13. Langer, O., Miodovnik, M., Reece, E. A., \& Rosenn, B. $M$. The proceedings of the diabetes in pregnancy study group of North America 2009 conference. Journal of Maternal-Fetal and Neonatal Medicine. 2010; 23(3), 196-198.

14. Hernandez-Rivas, E., Flores-Le Roux, J. A., Benaiges, D., Sagarra, E., Chillaron, J. J., Paya, A., ... \& Pedro-Botet, J. Gestational diabetes in a multiethnic population of Spain: Clinical characteristics and perinatal outcomes. Diabetes research and clinical practice. 2013. 100(2), 215-221.

15. Johns, K., Olynik, C., Mase, R., Kreisman, S., \& Tildesley, H. Gestational diabetes mellitus outcome in 394 patients. JOGC-TORONTO. 2006. 28(2), 122.

16. Chu, S. Y., Callaghan, W. M., Kim, S. Y., Schmid, C. H., Lau, J., England, L. J., \& Dietz, P. M. Maternal obesity and risk of gestational diabetes mellitus. Diabetes care. 2007; 30(8), 2070-2076.

17. Astudillo, D., Guillén, C., \& Gaybor, M. Prevalencia de parto por Cesárea en un Hospital de nivel III del Instituto Ecuatoriano de Seguridad Social. Rev Med HJCA. 2013, 5(1):12-16.

18. Ogonowski J., Miazgowski T. Intergenerational transmission of macrosomia in women with gestational diabetes and normal glucose tolerance. Eur J Obstet Gynecol Reprod Biol. 2015 Dec; (195):113-6

19. Gaudet L., Ferraro ZM., Wen SW., Walker M. Maternal obesity and occurrence of fetal macrosomia: a systematic review and meta-analysis. Biomed Res Int. 2014;2014:640291.

20. Hay Jr, W. W., Raju, T. N., Higgins, R. D., Kalhan, S. C., \& Devaskar, S. U. Knowledge gaps and research needs for understanding and treating neonatal hypoglycemia: workshop report from Eunice Kennedy Shriver National Institute of Child Health and Human Development. The Journal of pediatrics. 2009. 155(5).

21. DePuy, A. M., Coassolo, K. M., Som, D. A., \& Smulian, J. C. Neonatal hypoglycemia in term, nondiabetic pregnancies. American journal of obstetrics and gynecology. 2009. 200(5), e45-e51.

22. Flores-le Roux, J. A., Sagarra, E., Benaiges, D., Hernandez-Rivas, E., Chillaron, J. J., Puig de Dou, J., \& Pedro-Botet, J. A prospective evaluation of neonatal hypoglycaemia in infants of women with gestational diabetes mellitus. Diabetes research and clinical practice. 2012. 97(2), 217-222

23. Shin, Y.K., Kotelchuck, M., Wilson, H. G., Diop, H., Shapiro-Mendoza, C. K., \& England, L. J. (2015). Prevalence of Adverse Pregnancy Outcomes, by Maternal Diabetes Status at First and Second Deliveries, Massachusetts, 1998-2007. Preven- 
ting Chronic Disease. 2015 12, E218. htt//doi. org/10.5888/pcd12.150362

24. Gasim, T. Gestational diabetes mellitus: maternal and perinatal outcomes in 220 saudi women. Oman medical journal. 2012. 27(2), 140.

25. Bhat, M., Ramesha, K. N., Sarma, S. P., Menon, S., \& Ganesh Kumar, S. (2012). Outcome of Gestational Diabetes Mellitus from a Tertiary Referral Center in South India: A Case-Control Study. Journal of Obstetrics and Gynaecology of India, 62(6), 644-649.

26. Yessoufou, A., \& Moutairou, K. Maternal Diabetes in Pregnancy: Early and Long-Term Outcomes on the Offspring and the Concept of "Metabolic Memory.” Experimental Diabetes Research; 2011: 1-12.
27. Buchanan, T. A., \& Xiang, A. H. Gestational diabetes mellitus. The Journal of clinical investigation. 2005; 115(3), 485-491.

28. Carpenter, M. W. Gestational diabetes, pregnancy hypertension, and late vascular disease. Diabetes Care, 30 (Supplement 2). 2007. S246-S250.

29. Damm, P. Future risk of diabetes in mother and child after gestational diabetes mellitus. International Journal of Gynecology \& Obstetrics. 2009; 04, S25-S26.

30. Dabelea, D. The predisposition to obesity and diabetes in offspring of diabetic mothers. Diabetes care, 30(Supplement 2), 2007. S169-S174. 